\title{
Two-Week Treatment With the Selective Serotonin Reuptake Inhibitor Citalopram Reduces Contextual Anxiety but Not Cued Fear in Healthy Volunteers: A Fear-Potentiated Startle Study
}

\author{
Christian Grillon*,', Chanen Chavis', Matthew F Covington' and Daniel S Pine' \\ 'Mood and Anxiety Disorders Program, National Institute of Mental Health, National Institutes of Health, Bethesda, MD, USA
}

\begin{abstract}
Chronic treatment with selective serotonin reuptake inhibitors (SSRIs) alleviates both anxiety symptoms and associated physiologic disturbances in anxious patients. However, limited research considers the degree to which chronic SSRI treatment influences anxiety in healthy individuals. This study examined the effect of 2-week citalopram treatment on two threat responses: short- and long-durationpotentiated startle. Prior work suggests that these two responses provide neurally and functionally distinct models of fear and anxiety, respectively, in rodents. Healthy volunteers $(n=53)$ received either placebo or citalopram (20 mg per day) for 2 weeks under doubleblind conditions. They were each tested twice, before and after treatment. Participants were exposed to three conditions, including one in which predictable aversive shocks were signaled by a cue, a second in which unpredictable shocks were anticipated, and a third in which no shocks were administered. Aversive states were indexed by acoustic startle. Phasic fear-potentiated startle to the threat cue, as well as sustained startle potentiation to the experimental context in the predictable and unpredictable conditions, were investigated. Citalopram affected neither baseline startle nor short-duration fear-potentiated startle to discrete threat cues. However, citalopram reduced long-duration startle potentiation in the predictable conditions. These results are consistent with the hypothesis that short- and long-duration aversive states are mediated by distinct neural systems. They suggest that citalopram alleviates symptoms of anticipatory anxiety, not fear, by acting on mechanisms underlying long-duration aversive states.

Neuropsychopharmacology (2009) 34, 964-97I; doi: I0.1038/npp.2008.141; published online 17 September 2008
\end{abstract}

Keywords: fear; anxiety; SSRI; citalopram; startle reflex; predictability

\section{INTRODUCTION}

Growing evidence suggest that short- and long duration aversive states are functionally distinct (reviewed in (Grillon, 2008; Walker and Davis, 2008)). Studies based on the startle reflex suggest that the central nucleus of the amygdala (CeA) and the bed nucleus of the stria terminali (BNST) are involved, respectively, in phasic fear responses (CeA) to short-duration discrete cues and sustained anticipatory anxiety states to long-duration diffuse cues (BNST) (Walker and Davis, 2008). More specifically, phasic fear-potentiated startle to a discrete cue that signals a shock is mediated by the medial division of the CeA, whereas sustained potentiation of startle to more diffuse threat is

* Correspondence: Dr C Grillon, Mood and Anxiety Disorders Program, National Institute of Mental Health, NIH, I5K North Drive, Building I5K, Room II3, MSC 2670, Bethesda, MD 20892-2670, USA, Tel: + | 301594 2894, Fax: + | 3015949959 ,

E-mail: Christian.grillon@nih.gov

Received 28 June 2008; revised 5 August 2008; accepted 6 August 2008 mediated by projections from the basolateral amygdala and lateral CeA to the BNST (Walker and Davis, 2008). The hypothesis of a role for the BNST in sustained anxiety is consistent with results showing an involvement of the BNST in light-enhanced startle (Walker and Davis, 1997), context conditioning (Sullivan et al, 2004), Pavlovian fear conditioning with long-duration discrete cues (Waddell et al, 2006), and in the behavioral consequences of uncontrollable stress (Hammack et al, 2004).

If phasic and sustained threat responses reflect activity in dissociable neural systems, a pharmacological dissociation of these responses should be possible. Further, if sustained responses model anticipatory anxiety, one would expect these responses to be sensitive to medications that alleviate anticipatory anxiety in patients. We provided initial evidence for a pharmacological dissociation between phasic fear and sustained anxiety (Grillon et al, 2006). We showed that the benzodiazepine alprazolam did not affect fearpotentiated startle to a threat cue signaling a shock, but reduced the sustained potentiation of startle in contexts (ie contextual anxiety) in which shocks were administered 
(Grillon et al, 2006). These result, together with the recent finding that contextual anxiety but not cued fear is increased in panic disorder (Grillon et al, 2008), suggest that contextual anxiety models a clinically relevant phenomenon.

In the present study, we investigated the effects of the selective serotonin reuptake inhibitor (SSRIs) citalopram on cued fear and contextual anxiety. SSRIs are the medication of choice for anxiety, alleviating, among others, symptoms of anticipatory anxiety. A better understanding of the nature of aversive responses affected by SSRIs may have clinical implications and may help identify neural mechanisms of action of these drugs.

Little is currently known about the effect of SSRIs on experimental anxiety in humans. Most studies have examined the effect of a single dose of SSRIs (Harmer et al, 2003a; Kemp et al, 2004), but acute treatment may not inform on the anxiolytic effects of SSRI, which emerge only after chronic administration. In fact, SSRIs can be anxiogenic initially in patients (Gorman et al, 1987) and healthy volunteers (Grillon et al, 2007). Pharmacologically dissociating acute, anxiogenic from chronic anxiolytic effects in experimentally models would provide clinically relevant experimental data. However, experimental studies using chronic treatments are rare, due to the ethical and scientific hurdles confronting such research. Importantly, in one of the few available studies in this area, it was found that 7 days of citalopram (20 mg per day) impairs the recognition of facial expression of fear and anger (Harmer et al, 2004), reduces the modulation of startle by affective pictures (Harmer et al, 2004), and decreases amygdala responses to masked fearful facial expression (Harmer et al, 2006). However, the extent to which SSRIs affect emotional responses to more evocative threat cues remains unclear. Moreover, the degree to which these changes manifest with longer exposures more typical of clinical use also has been minimally explored.

Using the startle reflex, the present study examined the effect of 2 weeks of treatment with cilatopram on two types of responses to the threat of shock: fear associated with a discrete threat cue signaling a shock (cued fear-potentiated startle) and anticipatory anxiety experienced in the contexts (contextual anxiety-potentiated startle) in which the shocks are administered (Grillon et al, 2004, 2006). Two such contexts were implemented, one associated with predictable (ie signaled by a cue) shocks and the other with unpredictable (ie not signaled) shocks. Note that context, as defined here, refers to the background features presented during a specific experimental condition (Otto and Poon, 2006). It was expected that citalopram would reduce contextual anxiety. This hypothesis was based on the fact that SSRIs alleviate symptoms of anticipatory anxiety in anxious patients (Romano et al, 2004) and reduce risk assessment, a model of anticipatory anxiety, in mice (Blanchard et al, 2003). Existing data on cued fear generate competing hypotheses. Animal studies suggest that chronic citalopram can reduce cued fear conditioning (Burghardt et al, 2004), suggesting chronic citalopram may also reduce phasic fear in humans. On the other hand, data on experimental anxiety in humans, as noted above, suggest that benzodiazepines target long-duration as opposed to short-duration aversive responses. Similarly, prior clinical data on SSRI treatment suggest that clinically relevant treatments do not target fear-related symptoms (ie simple phobia) (Baldwin et al, 2005). However, no prior data examines experimentally induced cued fear in humans.

\section{METHODS}

\section{Participants}

Participants were paid healthy volunteers who gave written informed consent approved by the NIMH Human Investigation Review Board. Inclusion criteria included (1) no past or current psychiatric disorders as per Structured Clinical Interview for DSM-IV (SCID; First et al, 1995), (2) no history of mania in any first-degree relatives; (3) no medical condition that interfered with the objectives of the study as established by a physician, and (4) no use of elicit drugs or psychoactive medications as per urine screen. All potential participants met with a psychiatrist before providing consent. The full details of potential risks, including the risk for suicidal ideation, were discussed with each subject. They were randomized to the placebo or citalopram group. A total of 28 subjects (19 men) with a mean age of 28.8 years $(\mathrm{SD}=6.9$ years) were included in the placebo group. A total of 28 subjects also were included in the citalopram group. However, three subjects did not take the medication (see below). Their results were not included in the analysis. The final citalopram group consisted 25 subjects ( 15 men) with a mean age of 26.8 years ( $\mathrm{SD}=5.4$ years).

\section{Drugs}

A double-blind between-group pre-/post-treatment design was implemented in which one group received placebo and the other citalopram. Subjects were randomized to receive placebo for 14 days or citalopram at the dose of $10 \mathrm{mg}$ per day for 2 days followed by $20 \mathrm{mg}$ per day for 12 days. To ensure that subjects took the drugs blood levels of 5hydroxytryptophan, the precursor of serotonin, were examined at the conclusion of the study. Following the study, subjects were tapered over 4 days to $10 \mathrm{mg}$ per day. Study medication was then discontinued.

\section{Procedure}

Subjects were tested twice, once before and once after treatment. Subjects were seen by a psychiatrist at both the start and the end of the study. Phone contact was also maintained between these visits as well as in the week following the tapering of study medication. The two testing sessions were identical, except that at the end of the first session, subjects were given the placebo or citalopram pills to take every morning for the following 14 days. The second testing session was scheduled on the day of the last pill.

Each session started with a block of nine startle stimuli delivered every $18-25 \mathrm{~s}$ to reduce initial startle reactivity and to assess the effect of treatment on baseline startle. Afterwards the shock workup procedure was initiated, setting the shock intensity at a level that was highly annoying but not painful. All the subjects received three shocks during this period. After $15 \mathrm{~min}$, participants were given precise instructions regarding the conditions under 
which they would and would not receive a shock. The experiment consisted of three different conditions (Figure 1), a no shock condition (N), and two conditions during which shocks were administered either predictably $(\mathrm{P})$, that is, only in the presence of a threat cue, or unpredictably (U). Each condition lasted approximately $150 \mathrm{~s}$. In each 150-s condition, an 8-s cue was presented four times. The cues consisted of different geometric colored shapes for the different conditions (eg blue square for $\mathrm{N}$, red circle for $\mathrm{P}$ ). The cues signaled the possibility of receiving a shock only in the $\mathrm{P}$ condition, but had no signal value in the $\mathrm{N}$ and $\mathrm{U}$ conditions. Participants were verbally instructed regarding the risk of shock in the different conditions and they were informed of the contingency or lack of contingency between shock and cues in the $\mathrm{P}$ and $\mathrm{U}$ conditions. In addition, instructions were also displayed on a computer monitor throughout the experiment displaying the following information: 'no shock' (N), 'shock only during shape' $(\mathrm{P})$, or 'shock at any time' (U). During each $\mathrm{P}$ and $U$ condition, one shock was administered, during the cue in the $\mathrm{P}$ condition and in the absence of the cues in the $\mathrm{U}$ condition. In each $\mathrm{N}, \mathrm{P}$, and $\mathrm{U}$ condition, six acoustic startle stimuli were delivered, three during intertrial intervals (ITIs; ie, between cues) and one during three of the four cues, $5-7 \mathrm{~s}$ following cue onset. The threat experiment consisted of two recording blocks with a 510 min rest between blocks. Each block started with the delivery of six startle stimuli (pretest startle) and consisted of three $\mathrm{N}$, two $\mathrm{P}$, and two $\mathrm{U}$ conditions in one of the following two orders: P N U N U N P or U N P N P N U. Each participant was presented both of the orders, with half the participants starting with the $\mathrm{P}$ condition. One shock was administered in each individual $\mathrm{P}$ and $\mathrm{U}$ condition for a total of four shocks in the four P conditions and four shocks in the four $U$ conditions. The shock was delivered $7.5 \mathrm{~s}$ following cue onset in the $\mathrm{P}$ condition. It was administered either 7 or $10 \mathrm{~s}$ following cue offset in the unpredictable condition. No startle stimuli could follow a shock by less than $10 \mathrm{~s}$.

During testing, the Spielberger's state portion of the statetrait anxiety inventory questionnaire (Spielberger, 1983) was administered three times, just after subjects' arrival, at the end of the first block, and just after the second block. In addition, after each recording block, subjects

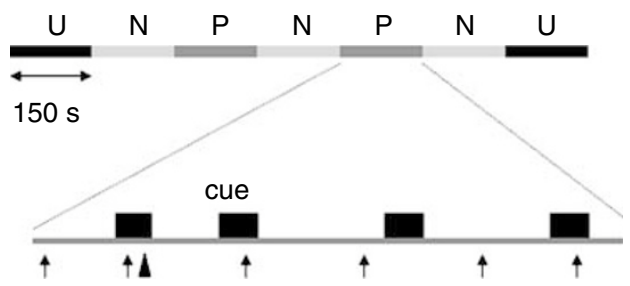

Figure I Schematic of the experiment. In one block, subjects were presented with three neutral $(N)$, two predictable $(P)$, and two unpredictable $(U)$ contexts (order $U N P N P N U$ as shown or P N U $N \cup N P$ ). Each subject was presented with two blocks, each associated with a different order. Each $N, P$, and $U$ condition contained four 8-s duration cues. In the $\mathrm{P}$ condition (as shown), the shocks were administered only in the presence of the cue. In the $U$ condition, the shocks were administered randomly in the absence of the cue. In the $\mathrm{N}$ condition, no shock was administered. The $\uparrow$ indicates a startle stimulus. The $\boldsymbol{\Delta}$ indicates a shock. retrospectively rated their anxiety level in the presence and absence of the cue in each condition $(\mathrm{N}, \mathrm{P}, \mathrm{U})$ on an analog scale ranging from 0 (not at all anxious) to 10 (extremely anxious).

\section{Stimuli and Physiological Responses}

Stimulation and recording were controlled by a commercial system (Contact Precision Instruments, London, UK). The acoustic startle stimulus was a 40 -ms duration, $103 \mathrm{~dB}$ (A) burst of white noise with a near instantaneous rise time presented binaurally through headphones. The eyeblink reflex was recorded with electrodes placed under the left eye. Amplifier bandwidth was set to $30-500 \mathrm{~Hz}$. The electric shock was produced by a constant current stimulator and administered on the left wrist.

\section{Data Analysis}

Peak amplitude of the startle/blink reflex was determined in the $20-100 \mathrm{~ms}$ time frame following stimulus onset relative to baseline (average baseline EMG level for the $50 \mathrm{~ms}$ immediately preceding stimulus onset) and averaged within each condition, after which they were standardized into $T$-scores. Fear was operationally defined as fear-potentiated startle during the discrete cues. Fear-potentiated startle scores were calculated as the difference between startle magnitudes during the cues minus startle magnitudes during ITI. Anxiety was operationally defined as anxietypotentiated startle. Anxiety-potentiated startle scores in the $\mathrm{P}$ and $\mathrm{U}$ conditions were calculated as the increase in ITI startle from the $\mathrm{N}$ to the $\mathrm{P}$ condition, and from the $\mathrm{N}$ to the $\mathrm{U}$ condition, respectively. The startle data and retrospective measures of subjective anxiety were analyzed with analyses of variance (ANOVAs) with repeated measures. For all statistical tests $\alpha$ was set at 0.05. Greenhouse-Geisser corrections (GG- $\varepsilon$ ) were used for main effects and interactions involving factors with more than two levels.

\section{RESULTS}

\section{Blood Levels of 5-Hydroxytryptophan}

The mean blood level of 5-hydroxytryptophan in the placebo group was $221 \mathrm{ng} / \mathrm{ml}(\mathrm{SD}=13 \mathrm{ng} / \mathrm{ml}$, range $=125$ $379 \mathrm{ng} / \mathrm{ml}$ ). Blood levels less than $50 \mathrm{ng} / \mathrm{ml}$ could not be detected. All subjects in the citalopram group had levels of $50 \mathrm{ng} / \mathrm{ml}$ or less, except the three excluded subjects who had levels in the range of the placebo group.

\section{Startle Magnitude}

Startle magnitudes during baseline and during the cues and ITI in the N, P, and $\mathrm{U}$ conditions pre- and post-treatments are shown in Table 1.

Baseline startle. Baseline startle (Table 1) was not affected by citalopram. A treatment group (placebo, citalopram $) \times$ session (pretreatment, post-treatment $) \times$ sex (men, women) ANOVA conducted on the baseline startle magnitude scores revealed no significant main effect or interaction effect (all $p>0.1$ ). 
Table I Mean (SEM) Startle Magnitude (T-scores) Before and After Treatment at Baseline, and During the Cue and ITI

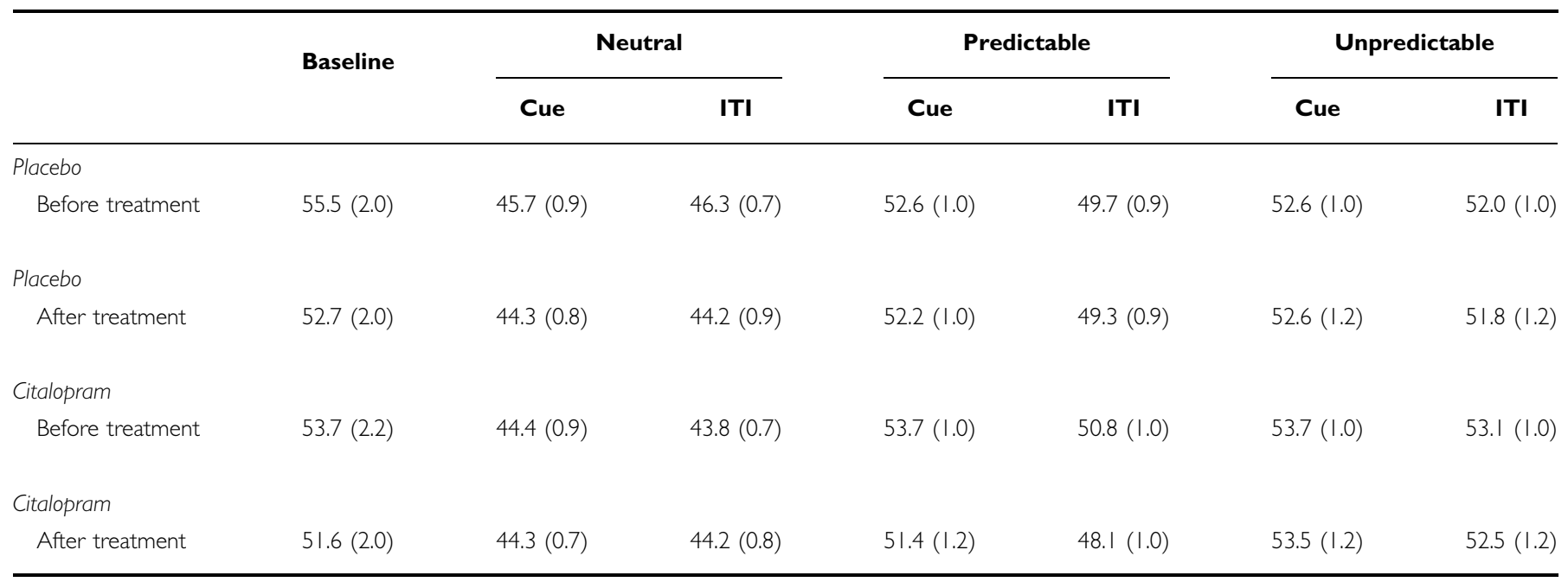

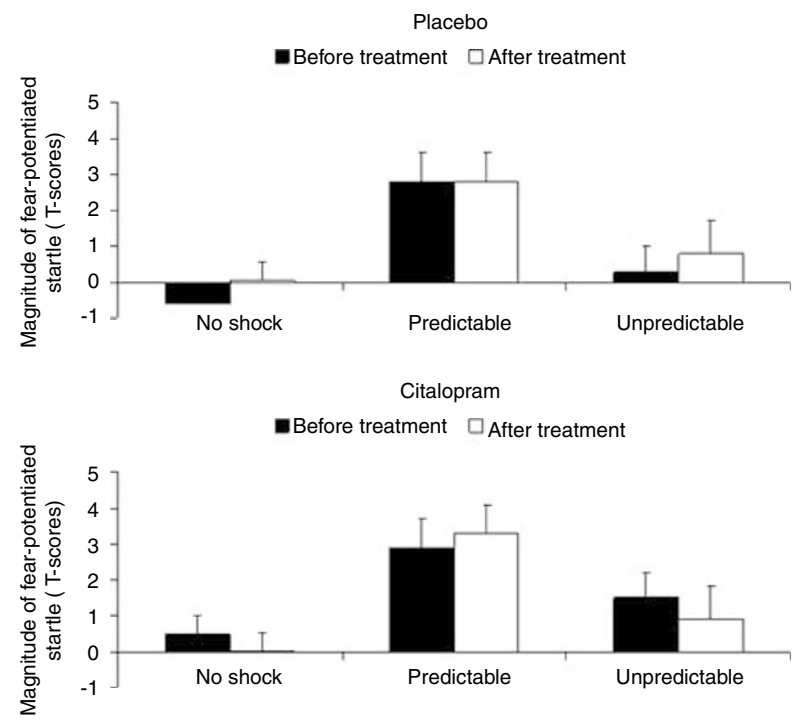

Figure 2 Fear-potentiated startle response to the cue in each treatment group before and after treatment: Difference scores reflecting cue minus ITI startle magnitudes in the neutral $(N)$, predictable $(P)$, and unpredictable $(U)$ conditions.

Fear-potentiated startle to the threat cue. The fearpotentiated startle scores were analyzed with a treatment group (placebo, citalopram) $\times \operatorname{sex}($ men, women $) \times$ session (pretreatment, post-treatment) $\times$ condition $(\mathrm{N}, \mathrm{P}, \mathrm{U})$ ANOVA. As expected, the magnitude of fear-potentiated startle was greater in the $\mathrm{P}$ condition compared to the $\mathrm{N}$ and $\mathrm{U}$ condition (Figure 2). This was confirmed by a condition main effect $(\mathrm{F}(2,98)=17.9, p<0.00001, \mathrm{GG}-\varepsilon=0.92)$ and a condition quadratic trend $(\mathrm{F}(1,49)=29.9, p<0.00009)$. This effect was not affected by citalopram as reflected by a nonsignificant treatment group $\times$ session $\times$ condition main effect or quadratic trend $(\mathrm{F}(2,98)=0.7, \quad \mathrm{NS}$ and $\mathrm{F}(1,49)=0.1$, NS, respectively). An analysis restricted to the predictable condition confirmed the lack of effect of citalopram on cued fear-potentiated startle (treatment group $\times$ session: $\mathrm{F}(1,49)=0.8, \mathrm{NS})$.

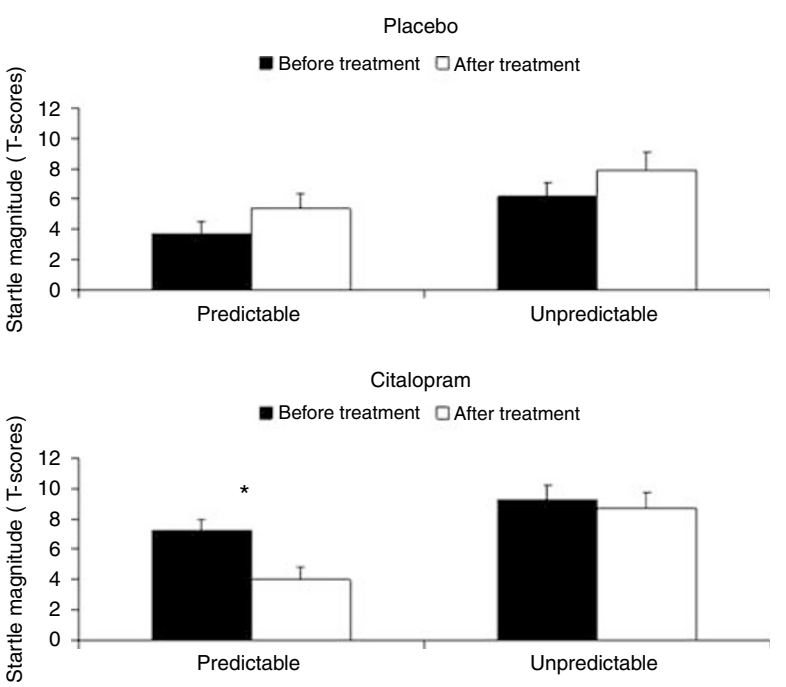

Figure 3 Anxiety-potentiated startle in each treatment group before and after treatment: Difference scores between ITI startle magnitudes in the threat conditions (predictable, $\mathrm{P}$, and unpredictable, $\mathrm{U}$ ) and ITI startle magnitude in the neutral $(\mathrm{N})$ condition. '*' indicates a significant $(p<0.05)$ reduction after treatment.

Anxiety-potentiated startle to the context. An initial analysis of the ITI startle data (Table 1) confirmed our previous results (Grillon et al, 2006) that startle magnitude increased progressively from the $\mathrm{N}$ to the $\mathrm{P}$ to the $\mathrm{U}$ condition. A treatment group (placebo, citalopram) $\times$ sex (men, women) $\times$ session (pretreatment, post-treatment $) \times$ condition $(\mathrm{N}, \mathrm{P}, \mathrm{U})$ ANOVA confirmed this effect (condition linear trend: $(F(1,49)=160.2, p<0.0001)$. Startle during ITI was differentially affected by citalopram and placebo as reflected by a significant treatment groupsession $\times$ condition $(\mathrm{F}(2,98)=3.3, \quad p<0.04, \quad \mathrm{GG}-\varepsilon=0.96)$ and treatment group $\times$ session $\times$ condition quadratic trend $(F(1,49)=6.2, p<0.02)$. Figure 3 , which shows the anxietypotentiated startle data (difference scores for ITI startle between $\mathrm{P}$ and $\mathrm{N}$, and $\mathrm{U}$ and $\mathrm{N}$ ), indicates that anxietypotentiated startle was slightly increased after treatment in 
Table 2 Mean (SEM) Retrospective Rating of Anxiety During the Cue and ITI Across Treatments and Conditions

\begin{tabular}{|c|c|c|c|c|c|c|}
\hline & \multicolumn{2}{|c|}{ Neutral } & \multicolumn{2}{|c|}{ Predictable } & \multicolumn{2}{|c|}{ Unpredictable } \\
\hline & Cue & ITI & Cue & ITI & Cue & ITI \\
\hline \multicolumn{7}{|l|}{ Placebo } \\
\hline Pretreatment & $1.9(0.2)$ & $1.8(0.2)$ & $5.4(0.4)$ & $3.6(0.4)$ & $5.1(0.4)$ & $5.4(0.4)$ \\
\hline Post-treatment & $1.4(0.1)$ & $1.4(0.1)$ & $5.5(0.4)$ & $3.2(0.3)$ & 5.5. (0.4) & $5.6(0.4)$ \\
\hline \multicolumn{7}{|l|}{ Citalopram } \\
\hline Pretreatment & $1.7(0.2)$ & $1.7(0.3)$ & $5.9(0.4)$ & $3.6(0.4)$ & $5.9(0.5)$ & $6.4(0.4)$ \\
\hline Post-treatment & $1.4(0.1)$ & $1.4(0.1)$ & $5.0(0.4)$ & $2.9(0.3)$ & $5.6(0.4)$ & $5.4(0.4)$ \\
\hline
\end{tabular}

the placebo group, but it was reduced in the citalopram group, suggesting an anxiolytic effect of citalopram, especially in the $\mathrm{P}$ condition. These observations were confirmed statistically. Treatment group $\times$ session ANOVAs on the anxiety-potentiated startle scores showed reduced contextual potentiation of startle in the citalopram group in the $\mathrm{P}$ condition (treatment group $\times$ session interaction: $\mathrm{F}(1,51)=8.3, p<0.006)$, whereas the reduction in the $\mathrm{U}$ condition failed to reach significance $(\mathrm{F}(1,51)=2.4$, $p=0.12$ ). Further, the reduction of startle potentiation in the $\mathrm{P}$ condition by citalopram was confirmed by withingroup analyses that showed a significant session $\times$ condition interaction for the $\mathrm{N} v s \mathrm{P}$ condition comparison in the citalogram group $(\mathrm{F}(1,24)=6.0, p<0.02)$, but not the placebo group $(\mathrm{F}(1,24)=2.2$, NS).

Women showed greater anxiety-potentiated startle compared to men $(\mathrm{F}(2,98)=4.0, p<0.04, \mathrm{GG}-\varepsilon=0.87)$, but this association was not moderated by citalopram $(\mathrm{F}(2,98)=0.8, \mathrm{NS}, \mathrm{GG}-\varepsilon=0.87)$. The mean startle potentiation $T$-score from the no shock to the $\mathrm{P}$ and $\mathrm{U}$ conditions (averaged together) was 6.5 $(\mathrm{SEM}=0.9)$ and $5.6(\mathrm{SEM}=1.1)$ in women and men, respectively.

\section{Retrospective Ratings of Anxiety}

The subjective anxiety ratings were analyzed in analogous manner as the startle data.

Cued fear. The retrospective anxiety rating scores (Table 2) were analyzed in the same way as the fear-potentiated startle data, ie using difference scores. Subjective anxiety to the cues (relative to ITI) was greater in the P condition compared to the $\mathrm{N}$ and $\mathrm{U}$ condition (condition main effect: $\mathrm{F}(2,98)=85.3, p<0.0001, \mathrm{GG}-\varepsilon=0.71)$. This effect was not affected by citalopram as reflected by a nonsignificant treatment group $\times$ session $\times$ condition $(F(2,98)=1.5$, NS). An analysis restricted to the $\mathrm{P}$ condition also did not reveal any effect of citalopram on subjective anxiety during the cue (treatment group $\times$ session, $\mathrm{F}(1,49)=1.9, \mathrm{NS})$.

Contextual anxiety. Like ITI startle amplitude, subjective anxiety (Table 2) increased linearly from the $\mathrm{N}$ to the $\mathrm{P}$ to the $U$ condition (condition linear trend: $F(1,49)=281.3$, $p<0.0001)$. The linear effect was less steep in the citalopram compared to the placebo group (treatment group $\times$
Table 3 Mean (SEM) State Anxiety at Three Time Points During Testing

\begin{tabular}{lccc}
\hline & Arrival & After first block & After second block \\
\hline $\begin{array}{l}\text { Placebo } \\
\text { Pretreatment }\end{array}$ & $26.6(1.3)$ & $30.9(1.7)$ & $28.6(1.6)$ \\
Post-treatment & $28.3(1.4)$ & $30.3(1.8)$ & $28.2(1.4)$ \\
& & & \\
Citalopram & & & $29.1(1.6)$ \\
Pretreatment & $27.3(1.3)$ & $31.3(1.7)$ & $28.2(1.4)$ \\
Post-treatment & $27.4(1.8)$ & $30.0(1.7)$ & \\
\hline
\end{tabular}

session $\times$ condition $\times$ treatment interaction linear trend: $\mathrm{F}(1,49)=4.1, p<0.05)$, reflecting a relative decrease in subjective anxiety in the citalopram group from the $\mathrm{N}$ to the $\mathrm{P}$ to the $\mathrm{U}$ condition. Subsequent analyses showed a trend for less anxiety in the citalopram group compared to placebo in the unpredictable $(\mathrm{F}(1,49)=3.8, p<0.06)$ but not the predictable $(\mathrm{F}(1,49)=0.1, \mathrm{NS})$ condition.

\section{State Anxiety}

The state anxiety scores (Table 3 ) were analyzed with a treatment group (placebo, citalopram) $\times \operatorname{sex}$ (men, women) $\times$ session (pretreatment, post-treatment $) \times$ time (baseline, after first block, after second block) ANOVA. There was only a significant main effect of time $(\mathrm{F}(2,98)=8.0, p<0.00001)$ and a time quadratic trend $(\mathrm{F}(1,49)=15.8, p<0.00001)$, reflecting a slight increase in anxiety after the first block. State anxiety after the first block was larger than at baseline $(F(1,52)=15.2$, $p<0.00001)$ or after the second block $(\mathrm{F}(1,52)=15.0$, $p<0.00001)$.

\section{DISCUSSION}

The present study used a psychopharmacological probe, citalopram, to establish a differentiation between shortduration and long-duration aversive states modeled by cued fear and contextual anxiety. The results show that a 2-week treatment with citalopram reduced contextual anxiety without affecting cued fear. The impetus for seeking a psychopharmacological differentiation between these two states comes from startle studies in rodents (Walker and Davis, 2008) and from empirical works in our laboratory. We have previously reported, using the same experimental design implemented in the present study, that cued fear is not affected by the benzodiazepine alprazolam (Grillon et al, 2006) and does not differ between healthy subjects and patients with panic disorder (Grillon et al, 2008). On the other hand, contextual anxiety is reduced by alprazolam (Grillon et al, 2008) and is elevated in patients with panic disorder, relative to healthy subjects (Grillon et al, 2008). These results are consistent with the hypothesis that longduration aversive states model anticipatory anxiety in anxious patients. 
There is evidence that SSRIs are efficacious in a number of anxiety disorders, but their mechanism of action is still largely unknown (Nutt et al, 1999). The present results suggest that citalopram does not affect CeA-mediated fear responses to a discrete threat cue. This is consistent with the clinical observation that fear-related symptoms such as specific phobias, respond less well to treatments such as citalopram than other pathological anxiety states, such as anticipatory anxiety in panic disorder (Baldwin et al, 2005). This is also consistent with preclinical evidence in rodents showing that the serotoninergic system is not implicated in the expression of cued fear-potentiated startle (Davis et al, 1988; Kehne et al, 1988). The findings that citalopram reduced contextual anxiety-potentiated startle is in line with the hypothesis that SSRIs have an 'antiworry' effect in anxious patients (Andrews et al, 1998). SSRIs also affect sustained anxiety states in animal models. In rodents, exposure to a cat litter, a model of sustained anxiety (Blanchard et al, 2001), produces a long-lasting increase in baseline startle that is suppressed by chronic sertraline (Matar et al, 2006). It is too early to speculate on the mechanisms responsible for the reduction of anxietypotentiated startle by citalopram. However, given the involvement of the stress hormone corticotrophin releasing factor (CRH) in the BNST in the mediation of the longduration potentiation of startle (Walker and Davis, 2008), it is possible that reduction in persistent anxious states is dependent on an interaction between serotonin and CRH. Indeed, there is evidence of interactions between CRF and SSRIs. Fluoxetine reduce CRF in depressed patients (De Bellis et al, 1993) and tryptophan depletion, which reduces brain serotonin, has the opposite effect (Tyrka et al, 2004).

Although citalopram reduced context potentiation of startle, this effect reached significance only in the predictable condition. One potential interpretation of these findings is that citalopram promotes the processing of or reliance on safety cues, thereby, reducing the overgeneralization of fear. Indeed, the nature of contextual anxiety in the predictable and unpredictable conditions substantially differs. In the predictable condition, context is measured during periods of clearly identifiable safety; this reflects the fact that shock could be administered only during the threat cues; their absence clearly signals periods of safety (Seligman and Binik, 1977). Hence, contextual anxiety in the predictable condition reflects generalization of fear from the cue to the context. This in not the case, in contrast, for contextual anxiety in the unpredictable condition, where contexts involves the actual risk of shocks, which can occur at any time. Therefore, citalopram's greater efficacy on contextual anxiety in the predictable, as opposed to the unpredictable, condition may reflect a reduction in the generalization of fear. This hypothesis is consistent with results of a knockout mouse model of anxiety involving the serotonin system (Klemenhagen et al, 2006). Mice lacking the serotonin $1 \mathrm{~A}$ receptor (5-HT1AR KO) exhibit increased anxiety-like behaviors (Parks et al, 1998). These mice show a strong tendency to overgeneralize fear, especially under conditions of competing safety and threat cues (Klemenhagen et al, 2006). One possibility is that SSRIs make safety cues more salient, reducing overgeneralization of fear. Of note, reminiscent of the present lack of effect of citalopram on cued fear, mice lacking the serotonin $1 \mathrm{~A}$ receptor do not show abnormal responses to a discrete conditioned fear cue (Klemenhagen et al, 2006).

SSRIs are used to treat a wide range of mood and anxiety disorders, including panic disorder. Although the present study showed an effect of citalopram on contextual anxiety in the predictable condition but not in the unpredictable condition, we recently showed that panic disorder patients have exaggerated contextual anxiety in the unpredictable condition, but not in the predictable condition (Grillon et al, 2008). It is premature to speculate on the implication of these apparent inconsistencies. We used milder aversive stimuli in our panic disorder study (eg loud sounds, screams) compared to the present study, which used shocks. Because different types of aversive stimuli generate different levels of contextual anxiety (Grillon et al, 2004), the two studies may not be directly comparable. In addition, the effect of SSRIs may be quite different in anxious patients compared to healthy individuals as suggested by studies with 5-HT1AR KO mice. These studies demonstrated different behavioral and anxiety responses to SSRIs in 5HT1AR KO and control mice (Malagié et al, 2002; Klemenhagen et al, 2006). Future studies should investigate whether SSRIs affect contextual anxiety in anxious patients (eg panic disorder), and whether this effect, if any, is associated with improvement in anticipatory anxiety symptoms.

The present results need to be interpreted in the context of its limitations. One limitation was that only a 2 -week treatment was implemented. Although this is longer exposure than in prior studies among healthy volunteers (Harmer et al, 2004, 2006), data in patients suggest that clinical benefits can continue to accrue over many weeks. Hence, longer exposures would be advantageous, though ethical questions could be raised on the appropriateness of exposing healthy individuals to SSRIs for longer than 2 weeks. Regardless, it should be noted that, consistent with the clinical observation that SSRIs can be anxiogenic in patients following initial treatment, we reported an increase in cued fear and contextual anxiety in a similar study using a single 20-mg dose of citalopram (Grillon et al, 2007). Taken together, the current and prior results demonstrate a dissociation between the acute and chronic effects of SSRIs on two types of experimentally induced anxiety. As such, these data demonstrate that our model is sensitive to the anxiogenic and anxiolytic effects of citalopram. Given the parallels between these findings and effects in clinical anxiety, one might suspect longer exposures in healthy subjects, as in patients, would produce steadily increasing anxiolytic effects on contextual anxiety; such sustained exposure also might potentially produce an effect on cued fear. Regardless, one main conclusion emerging from the current work is that a dissociable effect of chronic citalopram manifests on cued fear and contextual anxiety.

A second limitation was that the citalopram group had larger contextual anxiety compared to the placebo group (predictable and unpredictable context: $\mathrm{F}(1,51)=12.09$, $p<0.001$ and $F(1,51)=6.09, p<0.01)$. The impact of these differences on the results is unclear. Greater pretreatment contextual anxiety (ie greater ITI startle) in the predictable context could potentially affect the magnitude of fearpotentiated startle to the threat cue because of a ceiling effect. However, this is unlikely as startle magnitude of 
much larger magnitude compared to fear-potentiated startle were obtained during the initial habituation trials (data not show). The group difference in contextual anxiety also raises the question as to whether the decline in contextual anxiety in the predictable condition from pre-treatment to post-treatment in the citalopram group is due to regression to the mean rather than to a genuine reduction in anxiety. Although this is a possibility to consider, it is unclear why the citalopram group also did not experience a similar regression to the mean in the unpredictable context.

A final limitation was that subjective anxiety data did not fully match the startle findings. The subjective anxiety suggested that the anxiolytic effect of citalopram on contextual anxiety was stronger in the unpredictable condition, whereas the startle data suggest a stronger effect in the predictable condition. However, it is important to note that the startle data and the subjective reports converged in indicating an effect of citalopram on contextual anxiety but not on cued fear. In addition, reports of dissociation between objective measures and subjective reports are frequent. A single dose of citalopram increases the recognition of fearful faces without affecting subjective mood (Harmer et al, 2003b). Citalopram also affects physiological responses to emotional stimuli in the absence of conscious changes in subjective feelings (Kemp et al, 2004). We also reported that acute citalopram increased fear-potentiated startle to a threat cue and anxietypotentiated startle without affecting subjective fear/anxiety (Grillon et al, 2007). The most likely reason for the differential effect of citalopram on physiological and subjective reports in the present study is that startle was used to probe anxiety on-line, whereas the subjective anxiety measures were retrospective. The passage of time may have obscured subtle differences in responding because of the complexity of the design.

To summarize, consistent with the hypothesis of different neural mechanisms underlying fear-potentiated startle to a threat cue and context-potentiated startle, we found that 2 weeks of treatment with citalopram reduced the latter response without affecting the former. Using a similar experiment, we recently reported that patients with panic disorder (who are characterized by substantial levels of anticipatory anxiety) show normal fear-potentiated startle but elevated anxiety-potentiated startle. These findings confirm the relevance of contextual anxiety to clinical anxiety and have implications for psychopharmacological treatments. The demonstration of a pharmacological dissociation between fear and anxiety may encourage future efforts to develop novel treatments that target clinical manifestations of dissociable perturbations in these systems. For example, such treatments might relate to clinically relevant perturbations in so-called 'fear-related' systems, as manifest in simple phobia, or 'anxiety-related' systems, as manifest in anticipatory anxiety in panic disorder. There is growing evidence from animal models for the involvement of CRH on the BNST in mediating sustained startlepotentiated responses, and for interactions between serotonin and CRF in alleviating sustained anxiety states. Context-potentiated startle is therefore a useful integrative model to gain a better understanding of the mechanisms underlying the therapeutic effects of SSRIs and to detect the efficacy of potential anxiolytic compounds.

\section{ACKNOWLEDGEMENTS}

This research was supported by the Intramural Research Program of the National Institute of Mental Health.

\section{DISCLOSURE/CONFLICT OF INTEREST}

The authors declare that, except for income received from the primary employer, no financial support or compensation has been received from any individual or corporate entity over the past 3 years for research or professional service and there are no personal financial holdings that could be perceived as constituting a potential conflict of interest. Dr Pine has received compensation for activities related to teaching, editing, and clinical care that pose no conflicts of interest.

\section{REFERENCES}

Andrews W, Parker G, Barrett E (1998). The SSRI antidepressants: exploring their 'other' possible properties. J Affect Disord 49: 141-144.

Baldwin DS, Anderson IM, Nutt DJ, Bandelow B, Bond A, Davidson JR et al (2005). Evidence-based guidelines for the pharmacological treatment of anxiety disorders: recommendations from the British Association for Psychopharmacology. J Psychopharmacol 19: 567-596.

Blanchard DC, Griebel G, Blanchard RJ (2001). Mouse defensive behaviors: pharmacological and behavioral assays for anxiety and panic. Neurosci Biobehav Rev 25: 205-218.

Blanchard DC, Griebel G, Blanchard RJ (2003). The mouse defense test battery: pharmacological and behavioral assays for anxiety and panic. Eur J Pharmacol 463: 97-116.

Burghardt NS, Sullivan GM, McEwen BS, Gorman JM, LeDoux JE (2004). The selective serotonin reuptake inhibitor citalopram increases fear after acute treatment but reduces fear with chronic treatment: a comparison with tianeptine. Biol Psychiatry 55: 1171-1178.

Davis M, Cassella JV, Kehne JH (1988). Serotonin does not mediate anxiolytic effects of buspirone in the fear-potentiated startle paradigm: comparison with 8-OH-DPAT and ipsapirone. Psychopharmacology 94: 14-20.

De Bellis MD, Gold PW, Geracioti TD, Listwak SJ, Kling MA (1993). Association of fluoxetine treatment with reductions in CSF concentrations of corticotropin-releasing hormone and arginine vasopressin in patients with major depression. Am J Psychiatry 150: 656-657.

First MB, Spitzer RI, Williams JBW, Gibbon M (1995). Structured Clinical Interview for DSM-V (SCID). American Psychiatric Association: Washington, DC.

Gorman JM, Liebowitz MR, Fyer AJ, Goetz D, Campeas RB, Fyer MR et al (1987). An open trial of fluoxetine in the treatment of panic attacks. J Clin Psychopharmacol 7: 329-332.

Grillon C (2008). Models and mechanisms of anxiety: evidence from startle studies. Psychopharmacology 199: 421-437.

Grillon C, Baas JMP, Pine DS, Lissek S, Lawley M, Ellis V et al (2006). The benzodiazepine alprazolam dissociates contextual fear from cued fear in humans as assessed by fear-potentiated startle. Biol Psychiatry 60: 760-766.

Grillon C, Baas JP, Lissek S, Smith K, Milstein J (2004). Anxious responses to predictable and unpredictable aversive events. Behav Neurosci 118: 916-924.

Grillon C, Levenson J, Pine DS (2007). A single dose of the selective serotonin reuptake inhibitor citalopram exacerbates anxiety in humans: a fear-potentiated startle study. Neuropsychopharmacol 32: 225-231. 
Grillon C, Lissek S, Rabin S, McDowell D, Dvir S, Pine DS (2008). Increased anxiety during anticipation of unpredictable but not predictable aversive stimuli as a psychophysiologic marker of panic disorder. Am J Psychiatry 165: 898-904.

Hammack SE, Richey KJ, Watkins LR, Maier SF (2004). Chemical lesion of the bed nucleus of the stria terminalis blocks the behavioral consequences of uncontrollable stress. Behav Neurosci 118: 443-448.

Harmer CJ, Bhagwagar Z, Perrett DI, Vollm BA, Cowen PJ, Goodwin GM (2003a). Acute SSRI administration affects the processing of social cues in healthy volunteers. Neuropsychopharmacology 28: 148-152.

Harmer CJ, Mackay CE, Reid CB, Cowen PJ, Goodwin GM (2006). Antidepressant drug treatment modifies the neural processing of nonconscious threat cues. Biol Psychiatry 59: 816-820.

Harmer CJ, Rogers RD, Tunbridge E, Cowen PJ, Goodwin GM (2003b). Tryptophan depletion decreases the recognition of fear in female volunteers. Psychopharmacology 167: 411-417.

Harmer CJ, Shelley NC, Cowen PJ, Goodwin GM (2004). Increased positive $v s$ negative affective perception and memory in healthy volunteers following selective serotonin and norepinephrine reuptake inhibition. Am J Psychiatry 161: 1256-1263.

Kehne JH, Cassella JV, Davis M (1988). Anxiolytic effects of buspirone and gepirone in the fear-potentiated startle paradigm. Psychopharmacology 94: 8-13.

Kemp AH, Gray MA, Silberstein RB, Armstrong SM, Nathan PJ (2004). Augmentation of serotonin enhances pleasant and suppresses unpleasant cortical electrophysiological responses to visual emotional stimuli in humans. Neuroimage 22: 1084-1096.

Klemenhagen KC, Gordon JA, David DJ, Hen R, Gross CT (2006). Increased fear response to contextual cues in mice lacking the 5HT1A receptor. Neuropsychopharmacology 31: 101-111.

Malagié I, David DJ, Jolliet P, Hen R, Bourin M, Gardier AM (2002). Improved efficacy of fluoxetine in increasing hippocampal 5-hydroxytryptamine outflow in 5-HT(1B) receptor knockout mice. Eur J Pharmacol 443: 99-104.

Matar MA, Cohen H, Kaplan Z, Zohar J (2006). The effect of early poststressor intervention with sertraline on behavioral responses in an animal model of post-traumatic stress disorder. Neuropsychopharmacology 31: 2610-2618.

Nutt DJ, Forshall S, Bell C, Rich A, Sandford J, Nash J et al (1999). Mechanisms of action of selective serotonin reuptake inhibitors in the treatment of psychiatric disorders. Eur Neuropsychopharmacol 9(Suppl 3): S81-S86.

Otto T, Poon P (2006). Dorsal hippocampal contributions to unimodal contextual conditioning. J Neurosci 26: 6603-6609.

Parks CL, Robinson PS, Sibille E, Shenk T, Toth M (1998). Increased anxiety of mice lacking the serotonin 1A receptor. Proc Natl Acad Sci USA 95: 10734-10739.

Romano P, van Beek N, Cucchi M, Biffi S, Perna G (2004). Anxiety sensitivity and modulation of the serotonergic system in patients with PD. J Anxiety Disord 18: 423-431.

Seligman MEP, Binik YM (1977). The safety signal hypothesis. In: Davis H, Hurwitz HMB (eds). Operant-Pavlovian Interactions. Hillsdale: New York. pp 165-187.

Spielberger CD (1983). Manual for the State-Trait Anxiety Inventory. Consulting Psychologist Press: Palo Alto, CA.

Sullivan GM, Apergis J, Bush DE, Johnson LR, Hou M, Ledoux JE (2004). Lesions in the bed nucleus of the stria terminalis disrupt corticosterone and freezing responses elicited by a contextual but not by a specific cue-conditioned fear stimulus. Neuroscience 128: 7-14.

Tyrka AR, Carpenter LL, McDougle CJ, Kirwin PD, Owens MJ, Nemeroff CB et al (2004). Increased cerebrospinal fluid corticotropin-releasing factor concentrations during tryptophan depletion in healthy adults. Biol Psychiatry 56: 531-534.

Waddell J, Morris RW, Bouton ME (2006). Effects of bed nucleus of the stria terminalis lesions on conditioned anxiety: aversive conditioning with long-duration conditional stimuli and reinstatement of extinguished fear. Behav Neurosci 120: 324-336.

Walker D, Davis M (1997). Double dissociation between the involvement of the bed nucleus of the stria terminalis and the central nucleus of the amygdala in startle increases produced by conditioned $v s$ unconditioned fear. J Neurosci 17: 9375-9383.

Walker DL, Davis M (2008). Role of the extended amygdala in short-duration $v s$ sustained fear: a tribute to Dr Lennart Heimer. Brain Struct Funct 213: 29-42. 